\title{
LAM cells biology and lymphangioleiomyomatosis
}

\author{
Irmina Grzegorek ${ }^{1}$, Katarzyna Drozdz ${ }^{2}$, Marzenna Podhorska-Okolow ${ }^{1}$, \\ Andrzej Szuba ${ }^{2,3}$, Piotr Dziegiel ${ }^{1,4}$
}

\author{
1Department of Histology and Embryology, Wroclaw Medical University, Wroclaw, Poland \\ 2Department of Internal Medicine, 4-th Military Hospital, Wroclaw, Poland \\ 3Department of Internal Medicine, Wroclaw Medical University, Wroclaw, Poland \\ 4Department of Physiotherapy, Wroclaw University School of Physical Education, Wroclaw, Poland
}

\begin{abstract}
Progressive lung tissue destruction in lymphangioleiomyomatosis (LAM) occurs as a result of excessive proliferation of LAM cells caused by a mutation in one of the tuberous sclerosis complex suppressor genes, TSC1 or TSC2. These cells show constitutive activation of the mammalian target of rapamycin (mTOR) pathway and many of the mTOR-related kinases such as Akt, Erk, S6K1 and S6. Phenotype of LAM cells differs considerably depending on their microenvironment. LAM cells show differences in morphology, size and expression of various factors depending on their location in the tumor or body fluids. The presence of LAM cells in blood, urine, bronchoalveolar lavage fluid (BALF), and chyle proves their ability to metastasis. Antigens of smooth muscle cells are expressed in most LAM cells. Some of these cells are immunoreactive with HMB-45 antibody, which is used for the immunohistochemical diagnosis of LAM. Receptors for estrogen and progesterone may also be expressed in these cells, which probably is associated with the fact that LAM occurs almost exclusively in women of childbearing age. LAM cells via increased production of metalloproteinases are involved in the destruction of the extracellular matrix, as well as the remodeling and damage of lung tissue. Sporadic LAM occurs extremely rarely. Therefore a good experimental model of this disease is necessary. To date, several animal and human cell lines, which both genetically and phenotypically resemble LAM cells, have been obtained. These cell lines, derived from LAM nodule or an angiomyolipoma, are usually characterized by a mutation of the TSC2 gene, expression of smooth muscle cell antigens such as a-smooth muscle actin $(\alpha \mathrm{SMA})$ or S6K1 and S6 protein hyperphosphorylation. Presently, there is no commercially available cell line representing a good model of LAM. A better understanding of LAM cell biology is necessary for creating a useful model in vitro for further exploration of both LAM pathomechanisms and more general mechanisms of carcinogenesis. (Folia Histochemica et Cytobiologica 2013, Vol. 51, No. 1, 1-10)
\end{abstract}

Key words: lymphangioleiomyomatosis, LAM cells, mTOR, TSC1, TSC2, HMB-45, S6K1, $\alpha$ SMA

\section{Abbreviations}

4E-BP1 - eukaryotic translation initiation factor 4E-binding protein 1; AKT — protein kinase B; BALF — bronchoalveolar lavage fluid; EGF — epidermal growth factor; EGFR - epidermal growth factor receptor; elF4E - eukaryotic initiation factor-like protein; EMMPRIN — extracellular matrix metallopro-

Correspondence address: I. Grzegorek, Department of Histology and Embryology, Wroclaw Medical University, Chalubinskiego St. 6a; 50-368 Wroclaw, Poland; tel.: +48 7178413 65; mobile: +48 501647 494; fax: +48 7178400 82; email: irminagrzegorek@o2.pl teinase inducer; ER - estrogen receptor; ERK extracellular signal-regulated kinase; HIF-1 $\alpha$ - hypoxia-inducible factor 1a; IGF-1 — insulin-like growth factor 1; LAM - lymphangioleiomyomatosis; LOH - loss of heterozygosity; MAPK - mitogen-activated protein kinase; MART-1 - melanoma-associated antigen recognized by T cells; MEFs - mouse embryo fibroblasts; MMPs - matrix metalloproteinases; mTOR - mammalian target of rapamycin; PAI-1 - plasminogen activation inhibitor; PDK1 - phosphoinositide-dependent kinase-1; PGE2 - endogenous prostaglandin E2; PgR - progesterone receptor; PI3K - phosphoinositide 3-kinase; PLG - plasminogen; Rheb - Ras homolog enriched in brain; 

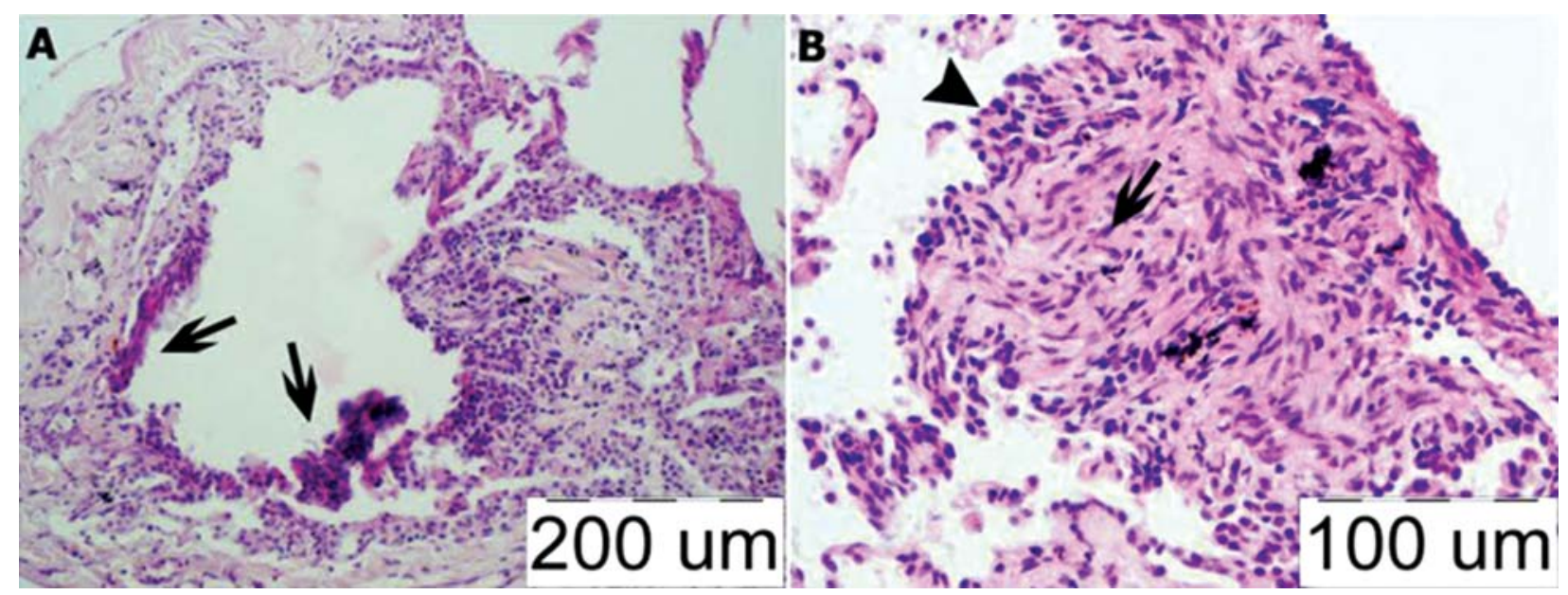

Figure 1. Surgical biopsy from 42-year-old woman with sporadic LAM (hematoxylin-eosin). (A) Lung tissue with thin walled cyst filled with LAM cells (arrows). (B) LAM nodule with spindle-shaped cells (arrows) in the central part of lesion and epithelioid-like cells at periphery of nodule (arrowhead)

RhoA - Ras homolog gene family, member A; S6K1 — ribosomal protein S6 kinase beta-1; SRF — serum response factor; STAT3 - signal transducer and activator of transcription 3; TIMPs - tissue inhibitors of matrix metalloproteinases; TORC1 - target of rapamycin complex 1; TORC2 - target of rapamycin complex 2; TRP - tyrosinase-related proteins; TSC - Tuberous Sclerosis Complex; uPA — urokinase-type plasminogen activator; aSMA — smooth muscle actin

\section{Introduction}

Sporadic pulmonary lymphangioleiomyomatosis (LAM) is a rare disease, affecting almost exclusively women. It is characterized by intense proliferation of smooth muscle-like cells (LAM cells) in the lungs and around bronchi, blood and lymphatic vessels, which leads to the formation of thin-walled cysts (Figure 1A), the degeneration and remodeling of lung tissue, progressive deterioration of lung function and eventually to death [1-3]. The incidence of sporadic LAM is estimated at 2.6 per 1 million women. LAM may also be associated with an autosomal dominant disease tuberous sclerosis complex (TSC) and occurs in approximately one-third of the women affected by TSC [3]. Hamartoma tumors in various organs such as skin, eyes, kidneys, lungs, and the central nervous system are present in TSC. Sporadic LAM may also give extrapulmonary symptoms, similar to those in TSC, such as renal angiomyolipomas, axial lymphadenopathy, and abdominal lymphangiomyomas [1]. LAM lesions are characterized by an infiltration and accumulation of smooth muscle-like cells. Mutations in either tumor suppressor gene TSC1 or TSC2 cause excessive proliferation of LAM cells and support their ability to metastasis. The phenotype of LAM cells, which include smooth muscle cells appearance with melanoma phenotype is a useful feature in LAM diagnosis [4].

\section{The genetics of LAM and the mTOR pathway}

TSC1 and TSC2 belong to the group of tumor suppressor genes, namely the tuberous sclerosis complex. The TSC1 gene is located on chromosome 9q34, and consists of 21 exons encoding 1164 amino acid protein hamartin. The TSC2 gene on chromosome 16p13 contains 41 exons encoding 1807 amino acid protein tuberin [5]. Hamartin and tuberin form a protein complex, which reduces the level of Rheb-GTP through activation of GTP-ase, resulting in an inhibitory effect on mammalian target of rapamycin (mTOR), a highly conserved serine-threonine kinase that plays an important role in the regulation of cell growth and proliferation. Growth factors, phosphoinositide 3-kinase (PI3K) and phosphoinositidedependent kinase1 (PDK1) dependent stimulation leads to the phosphorylation and activation of protein kinase $\mathrm{B}$ (Akt). Activated Akt, as well as extracellular signal-regulated kinase (ERK), phosphorylates TSC2 resulting in inhibition of its activity as a GTP-ase. Akt is known as an activator of mTOR and is involved in the pathogenesis of many cancer types. Mutation in one of the TSC genes causes the protein complex to be inactivated which promotes mTOR activation leading to the phosphorylation and activation of ribosomal protein S6 kinase beta-1 (S6K1) and S6 ribosomal subunit. This results in the activation of translational 
mechanisms, increased cell growth and proliferation (Figure 2) [6]. Active mTOR kinase phosphorylates eukaryotic translation initiation factor 4Ebinding protein 1 (4E-BP1) which inhibits its interaction with eukaryotic initiation factor-like protein (elF4E), resulting in the activation of translation and increased cell cycling (Figure 2) [7]. mTOR kinase is a component of two functional complexes: TORC1 and TORC2. TORC1 is the rapamycin-sensitive mTOR complex responsible for the regulation of protein translation initiation and efficiency [8]. Clinical trials with rapamycin as a therapeutic solution for lymphangioleiomyomatosis are in an advanced phase [9]. Rapamycin is a macrolide antibiotic isolated from a strain of Streptomyces higroscopicus. It has been already used as an immunosuppressive agent. Rapamycin binds to the cytosolic protein FKPB-12 thereby inactivating the mTOR kinase. Treatment with rapamycin resulted in the reduction of tumor volume and improved lung function in LAM patients [9-11]. However, rapamycin only partially inhibits cell growth, proliferation and disease progression. Yu et al.[8] presented two possible explanations of this fact which may coexist in human LAM. If rapamycin can cause a decrease of cell size and TORC1 inactivation, then a further decrease of tumor size could be obtained, for example, via the inhibition of TSC2-dependent TORC1/rapamycin independent pathway. The second hypothesis assumes that rapamycin can cause a decrease in cell number but some group of cells can still show the TORC1 activation caused by unequal drug delivery or development of rapamycin-resistance. It is hoped that tumor size could be further decreased by using other TORC1 inhibitors, developing a better system of drug delivery or overcoming rapamycin resistance [8].

LAM in women with TSC is associated with germline mutations in TSC1 or TSC2 genes. Depending on the type of TSC gene mutation differences in the incidence and severity of pulmonary symptoms have been observed. A significantly higher number of cysts in the lungs was detected in patients with mutations in the TSC2 gene compared to patients with TSC1 mutations [12]. It seems that, in particular, changes in the C-terminal segment of tuberin caused by mutations in exons 40-41 of the TSC 2 gene may be associated with LAM symptoms in patients with tuberous sclerosis [5]. Loss of heterozygosity (LOH) of one of the TSC genes in somatic cells and clonal expansion of these cells are responsible for sporadic LAM $[13,14]$. According to the „two hits” Knudson's theory two independent events lead to the loss of both functional copies of this gene [15]. Sporadic LAM is caused by mutations almost exclusively within the TSC2 gene. Germline mutations were not observed

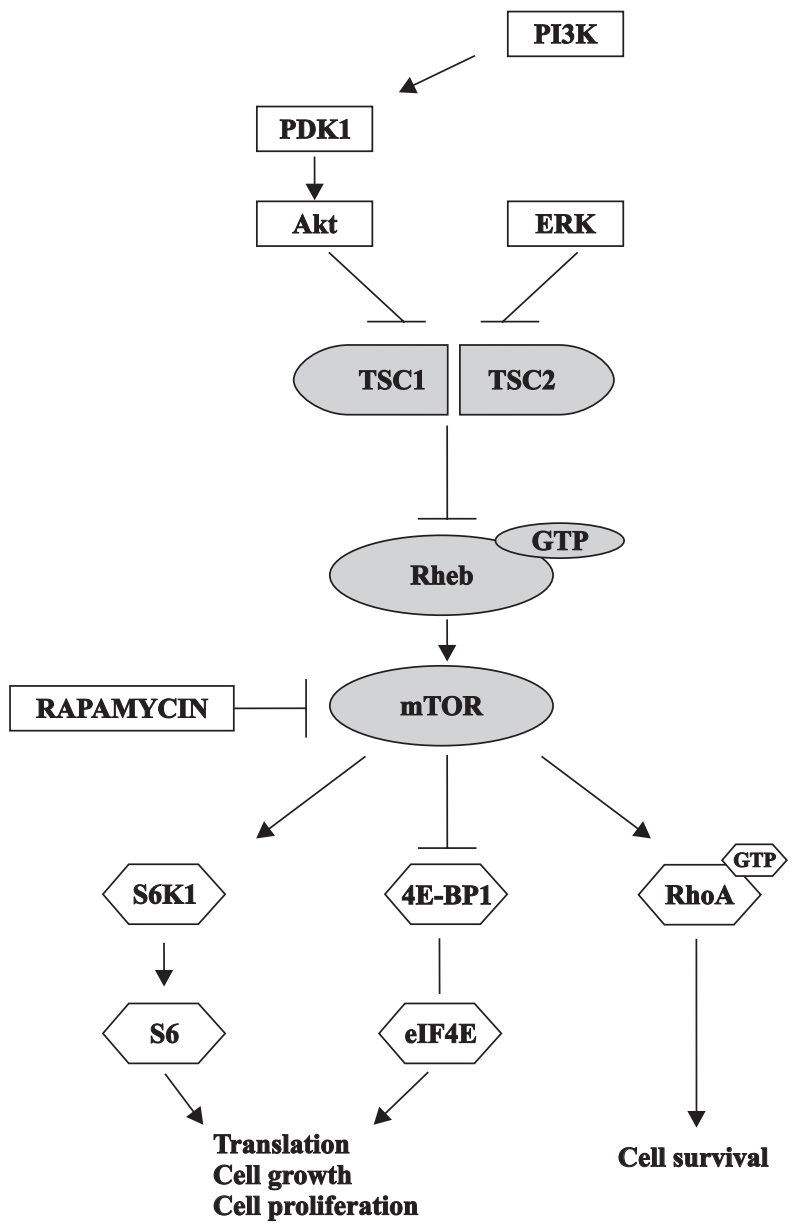

Figure 2. Schematic representation of mTOR signaling pathway. TSC1-TSC2 complex is involved in mTOR inhibition. Inactivation of this complex in LAM results in constitutive activation of mTOR and in an increase of LAM cells growth and proliferation. The mTOR may be also activated by PI3K - dependent activation of Akt leading to the inhibition of TSC complex. Phosphorylation of TSC2 by ERK leads to the inactivation of TSC complex. Activation of mTOR results phosphorylation and activation such proteins as $\mathrm{S} 6 \mathrm{~K} 1$ and RhoA leading to enhancement of translation, cells growth and proliferation as well as cells survival. Phosphorylation of 4E-BP1 by mTOR leads to inhibition of interaction between $4 \mathrm{E}-\mathrm{BP} 1$ and elF4E resulting in activation of translation and increase in cells proliferation. Rapamycin, a novel therapeutic solution in lymphangioleiomyomatosis, is a natural inhibitor of mTOR kinase. mTOR — mammalian target of rapamycin; TSC — tuberous sclerosis complex; PI3K - phosphoinositide 3-kinase; Akt - protein kinase B; ERK - extracellular signalregulated kinase; S6K1 — vRibosomal protein S6 kinase beta-1; RhoA - Ras homolog gene family, member A; 4E-BP1 - eukaryotic translation initiation factor 4E-binding protein 1; elF4E - eukaryotic initiation factor like protein.

[16]. There is a significant association between loss of TSC1/TSC2 function and an increase of LAM cells invasiveness and motility. Ras homolog gene family, 


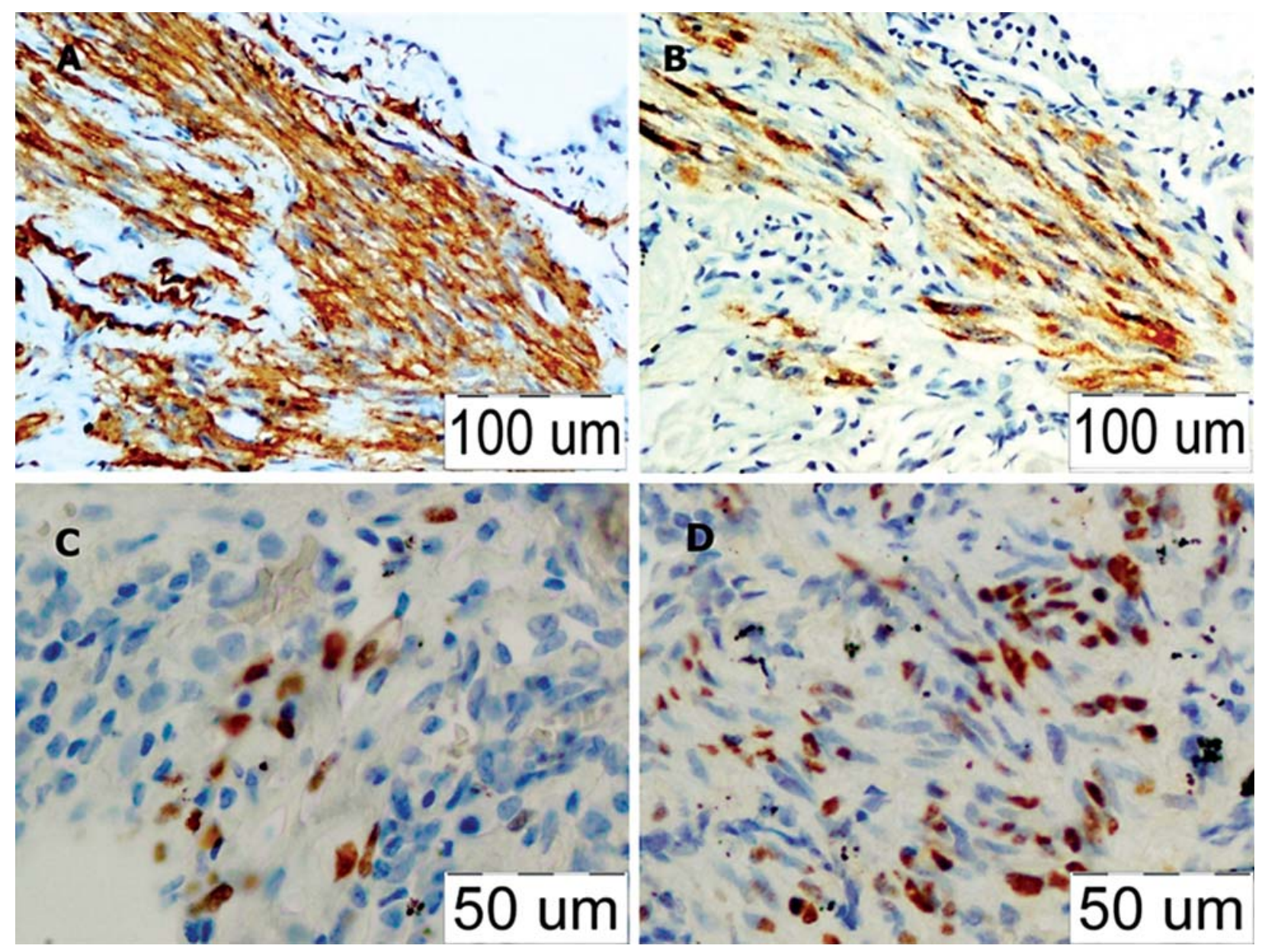

Figure 3. Surgical biopsy from 42-year-old woman with sporadic LAM (immunohistochemical staining). (A) $\alpha$-smooth muscle actin, $\alpha$-SMA; (B) HMB-45; (C) Estrogen receptor, ER, (D) Progesterone receptor, PgR

member A (RhoA) is a small GTPase protein known to regulate the actin cytoskeleton in the formation of stress fibers. RhoA activity is regulated by TSC1/TSC2 complex formation. TSC2 binds the TSC1 via its binding domain, which overlaps with the RhoA activating domain present in the protein encoded by the TSC1. TSC2 mutation results in the abnormal formation of the TSC1/TSC2 complex and an increase in RhoA activity which increases the invasiveness and migration ability of LAM cells [17]. It was shown that transfection of primary culture of human LAM cells by normal gene TSC2, as well as TSC1 gene silencing resulted in the inhibition of excessive RhoA activity. Loss of TSC2 function by the dysregulation of the TSC1/TSC2 complex formation leads to the TSC1dependent RhoA activation, an increase of invasiveness, migration, and thus the metastatic nature of LAM cells [17]. The ability to metastasis of $\mathrm{TSC}^{-1-}$ cells can be also explained by the presence of cleaved forms of $\beta$-catenin leading to an increased matrix metalloproteinase 7 (MMP7) expression and thus the invasiveness of these cells [18].

\section{LAM cell phenotype}

LAM cells are morphologically heterogeneous with a phenotype ranging from smaller spindle-shaped smooth muscle-like cells to larger epithelioid-like cells which are abundant with the cytoplasm. Smaller, spindle-shaped cells are located centrally, while epithelioid-like cells are observed mainly at the periphery of the LAM nodule (Figure 1B) [2, 19, 20]. The arrangement of these cells becomes more irregular as the disease progresses [20]. It was suggested that LAM cells proliferation occurs in the central part of the tumor, and then the cells grow intensively, differentiate and migrate to peripheral parts of the tumor [20].

LAM cells show phenotypic features of smooth muscle cells since they express smooth muscle actin $(\alpha \mathrm{SMA})$ (Figure 3A), vimentin and desmin [2,11, 20, 
21]. Ultrastructural studies showed the presence of filaments that react with antibody to smooth muscle F-actin, as well as intermediate desmin and vimentin filaments which were located around nuclei [20]. Expression of antigens specific to melanoma, and especially the reaction with the HMB-45 antibody (Figure 3B) is a useful tool for lymphangioleiomyomatosis diagnosis by immunohistochemistry [11, 20]. HMB45 is a mouse monoclonal antibody which reacts with gp100 glycoprotein, a product of alternative splicing of pmel17, characteristic for melanoma and LAM cells, especially for epithelioid-like cells of the latter [20]. Electron-dense granules resembling immature melanosomes in LAM cell cytoplasm were observed by electron microscopy. Further studies confirmed the immunoreactivity of these structures with the HMB45 antibody [22]. Some of the LAM cells also express a small transmembrane protein, melanoma-associated antigen recognized by T cells (MART-1), which is widely expressed in melanoma cells [23]. Both gp100 and MART- 1 can be recognized by T cells, increasing the susceptibility of these cells to the cytotoxic activity of T cells. This feature may become an important therapeutic target. Expression of tyrosinase-related proteins TRP-1 (gp-75) and TRP-2 was also observed in LAM cells [23]. Other markers of melanoma, such as CD63, a protein belonging to the tetraspanin family, or a recently described unknown protein which reacts with PNL2 antibody, are also present in LAM cells [24].

Due to the fact that LAM almost exclusively affects women, estrogen (ER) and progesterone (PgR) receptors were examined in LAM cells. The expression of both receptor types was observed in the nuclei of LAM cells (Figure 3 C, D). However, greater frequency and stronger reactivity was seen for PgR compared to ER [25, 26]. The expression of both receptors was observed mainly in larger epithelioid LAM cells and in some relatively larger spindle shaped cells [26]. Estrogen treatment of TSC2-null ELT3 (epithelioid leiomyoma tumor) cells led to increased cell proliferation in vitro and significantly enhanced the metastazing ability of these cells in vivo [27]. Although the first therapeutic trials were based on suppression of estrogen activity, the efficacy of these therapies has not yet been proven. Migration and behavior of LAM cells depends on the activities of specific chemokines. These cells, like other cancer cells, have a characteristic expression profile of chemokines and their receptors. High expression of CXCR4 receptor was detected in LAM cells. Loss of TSC2 function, through activation of mTOR, can lead to the increased expression of hypoxia-inducible factor $(\mathrm{HIF}-1 \alpha)$, which is a transcriptional factor for sev- eral genes during hypoxia, including CXCR4. The expression of some chemokines and their receptors may be partially regulated by hypoxia occurring in patients with moderate or highly advanced LAM. LAM cells also produce large amounts of CCL2. CCL2 and its receptors CCR2 and CCR10 probably play an important role in the pathogenesis of LAM. In vitro studies showed that in a heterogeneous population of cells CCL2 was a selective chemoattractant of LAM cells [28]. Interestingly, high expression of this chemokine was also observed in a dominant negative transgenic mouse model of TSC2 [28]. High expression of the chemokines CCL2, CXCL1 and CXCL5 in bronchoalveolar lavage fluid (BALF) of LAM patients as well as in cells derived from LAM nodules was observed. This may suggest their involvement in the pathogenesis of LAM [28]. Clements et al. [29] showed CCR3, CXCR4, CXCR6 and CXC3CR1 expression in angiomyolipoma and LAM. Ligands for these receptors (CXCL12 CX3CL1, CCL11, CCL24, and CCL28) are responsible for the phosphorylation of Akt and mitogen-activated protein kinase (MAPK), which promote the mTOR pathway. Interestingly, CXCL12, the only known ligand for CXCR4, was produced by type II pneumocytes surrounding the LAM nodules and vascular endothelium [29]. It may function as a chemoattractant and survival factor for circulating LAM cells. CCR1, CCR7, and CXCR7 mRNA were detected in angiomyolipoma primary cell culture [29]. Expression of the specific chemokine profile in LAM was shown to affect LAM cells migration, metastasis, tumor progression and interaction between LAM and stroma cells [28-30].

The ability to metastasis of LAM cells is also related to the production of proteolytic enzymes, for example, matrix metalloproteinases (MMPs). LAM cells showed expression of several metalloproteinases such as MMP-1, MMP-2, MMP-3, MMP-7, and MMP-9 [30]. Proteolysis of extracellular matrix components, including different types of collagen, proteoglycans and elastic fibers is regulated by the activity of MMPs and their tissue inhibitors (TIMPs). Hayashi et al. [31] demonstrated immunohistochemically the expression of MMP-1, MMP-2 and MMP-9 in LAM cells. An interesting finding was that morphologically different types of cells in the LAM nodule showed a different expression pattern of MMPs. Large LAM cells that were immunoreactive with the HMB-45 antibody showed a stronger reaction with MMP-2 and MMP-9 than other types of LAM cells in the nodule [31]. Elevated levels of Serum Response Factor (SRF) in LAM cells may be an important factor in the degradation of lung tissue. SRF is an essen- 
tial transcriptional factor which is highly expressed in developing smooth muscle cells, however, its expression decreases in mature cells [32]. LAM cells are morphologically similar to immature myoblasts and expression of SRF was observed in both nuclei and cytoplasm. High SRF level increased expression of MMP-2 and MMP-14 and reduced expression of one of their inhibitors, the metalloproteinase inhibitor 3 (TIMP-3) [32]. The lower level of TIMP-3 may be of particular significance in the progression of LAM since TIMP-3-null mice showed a destructive lung phenotype, which led to progressive emphysema and a shorter life span [32]. A study with the overexpression of MMPs in human lung fibroblasts demonstrated that high levels of SRF may also regulate the plasminogen system in vitro [33]. SRF, by increasing levels of urokinase-type Plasminogen Activator (uPA), activates plasminogen (PLG) to plasmin, which in turn activates MMPs. A high level of SRF reduces the expression of plasminogen activation inhibitor (PAI-1). These results [33] were confirmed by laser micro-dissection, RT-PCR, and immunohistochemistry on sections of lung tissue from LAM patients. Strong positive immunohistochemical reactions with uPA and PLG were observed in LAM nodules compared to the surrounding healthy lung tissue. PAI-1 expression was observed in normal lung tissue, whereas it was not present in LAM lesions [33]. Another protein which was shown to regulate the activity of MMPs, extracellular matrix metalloproteinase inducer (EMMPRIN/CD147), was detected in LAM cells. Double immunofluorescence revealed co-localization of EMMPRIN/CD147 and $\alpha$ SMA, MMP-2 or MMP-9. Levels of EMMPRIN/CD147 were significantly elevated in LAM lesions and BALF of LAM patients [34]. High expression of MMPs in TSC2 $2^{-/-}$angiomyolipoma cells seems to be independent of mTOR kinase and their levels do not change following rapamycin treatment, therefore the treatment of LAM by this mTOR inhibitor may have limited clinical effectiveness [35]. It has been hypothesized that doxycycline, as an inhibitor of MMPs, could inhibit degradation of lung tissue in LAM patients. Clinical trials have shown that administration of doxycycline to LAM patients resulted in lower levels of MMP-2, MMP-9 in serum and urine, decreased symptoms and improved life quality [36, 37]. However, in vitro studies showed that there was no significant reduction in the level and activity of MMPs in the ELT3 ${ }^{-/}$cells, derived from an animal model of LAM, the Eker rat, after doxycycline cells treatment [38]. Thus, the efficacy of doxycycline to inhibit MMPs activity in LAM patients needs to be further investigated.

Many of the above described features of LAM cells reveal their ability to metastasize. So far, the most useful marker of these cells, commonly used in the differential diagnosis of LAM is an immunohistochemical reaction with the antibody HMB-45. Exploring the biology of LAM cells, in particular characterization of disseminated neoplastic LAM cells in the body fluids, could lead to a development of new diagnostic tools which would be less invasive than a biopsy. In order to find specific markers for LAM cells circulating in blood, urine or BALF, PachecoRodriguez et al. [39] carried out a series of molecular studies. Analysis of blood samples from LAM patients showed that cells with TSC2 LOH were immunoreactive with anti-CD235a antibody against glycophorin-A, a sialoglicoprotein present in erythrocyte's cell membrane. The expression of this protein was also demonstrated in $31 \%$ of lung LAM cases. This protein was also reported to be present on the surface of breast cancer and melanoma cells [39]. It was also found that cells from the nodule express CD44v6, the product of CD44 alternative splicing [40]. CD44 is a class I transmembrane glycoprotein. The formation of CD44 isoforms is regulated by a signaling pathway involving the Ras-mitogen activated protein kinase pathway [40]. Isoforms generated by alternative splicing of 10 additional exons from v1 to v10 resulted in an insertion of additional segments in the extracellular domain of the protein. The extracellular domain of CD44 binds metalloproteinases such as MMP-7 and MMP-9, which cleave CD44 and CD44v6 [40]. CD44v6 is associated with carcinogenicity and is involved in homing during metastasis, and its expression was demonstrated on squamous cell carcinoma and adenocarcinoma cells [40]. The CD44v6 domain can also bind Fas ligand, which inhibits apoptosis. LAM lesions showed moderate reaction with CD44, however, the usefulness of this antigen for LAM cells isolation from body fluids is limited because it shows a strong immunoreactivity with other cell types such as vascular smooth muscles cells, bronchial epithelial cells and hyperplastic type II pneumocytes [40]. A large percentage of cells in the LAM nodule showed expression of CD44v6 in contrast to other cells in the lung tissue. CD44+/CD44v6+ cells from LAM nodule, exhibit LOH of the TSC2 gene [40]. The presence of CD44v6 was found on LAM cells isolated from BALF, urine, and chyle [41]. Microarray analysis performed on material derived from the skin tumor associated with TSC showed high expression of $\mathrm{CD} 9$, a protein of the tetraspanin family which plays an important role in cell morphology, motility, invasiveness, adhesion as well as cellular interactions [41]. Its expression may correlate with metastases of tumor cells. A higher level of CD9 was correlated with TSC2 LOH [41]. Both CD44v6 and CD9 are useful markers to distinguish and isolate LAM cells from 
Table 1. Mechanisms of metastasis in lymphangioleiomyomatosis

\begin{tabular}{|l|l|}
\hline Mutations and markers in LAM cells & Mechanisms \\
\hline Loss of heterozygosity of TSC2 & $\begin{array}{l}\text { Constitutive activation of mTOR pathway, enhanced cell growth and proliferation, } \\
\text { cells survival [13,14] }\end{array}$ \\
\hline Estrogen receptor, ER & $\begin{array}{l}\text { Estrogens promote proliferation, survival and lung colonization of ELT3 cells, } \\
\text { activation of MMPs [27] }\end{array}$ \\
\hline $\begin{array}{l}\text { Chemokines and its receptors e.g. } \\
\text { CCL2, CCR2, CXCR4 }\end{array}$ & Enhanced migration, homing, and interactions between LAM and stroma cells [28-30] \\
\hline $\begin{array}{l}\text { Metalloproteinases, MMPs e.g. } \\
\text { MMP-2, MMP-9 }\end{array}$ & Degradation of extracellular matrix which facilitates LAM cells invasion [27] \\
\hline CD44, CD44v6 & Homing, inhibition of apoptosis by binding Fas ligand [40] \\
\hline CD9 & Motility, invasiveness, adhesion, cells interactions [41] \\
\hline
\end{tabular}

body fluids. CD44v6+/CD9+ cells showed TSC2 LOH in $80 \%$ in BALF, $69 \%$ in urine and $50 \%$ in chyle [41]. Changes in metastatic cell phenotype can occur depending on the microenvironment. The LAM cells in different locations may exhibit different antigen expression patterns such as CD44/CD44v6 expression in LAM cells from the LAM nodule, CD235 in LAM cells in blood and CD44v6/CD9 expression in LAM cells in urine, BALF, and chyle [41].

Metastatic features of LAM cells are presented in Table 1 .

\section{Cell lines as a model of LAM}

Lymphangioleiomyomatosis is a rare interstitial lung disease. An applicable experimental model is still required for carrying out further studies. The most popular animal model of LAM is the Eker rat with TSC2 germline mutation occurring in one of its alleles. In this model rats develop renal adenomas, uterine leiomyomas and pituitary adenomas. In some of them loss of heterozygosity for the TSC2 gene may be observed. Heterozygous TSC $2^{+/-}$mice develop changes similar to those observed in the Eker rat, and some of them were characterized by TSC2 LOH. In $\mathrm{TSC}^{+/-}$ mice a similar phenotype was seen, however, kidney tumors developed with a lower frequency [42]. Germline inactivation of both alleles of TSC1 or TSC2 in mice is lethal [43].

The most commonly used cell lines derived from an animal model of LAM are TSC2-null lines derived from tumors present in the Eker rat, ELT 3, 4, 6, 9, 10. These lines, isolated from uterine leiomyomas, exhibit expression of smooth muscle antigens and constitutive activation of mTOR kinase. All of these cell lines express receptors for estrogen and progesterone, however, only ELT6 was shown to respond to these hormones in culture [44]. Although TSC1 and
TSC2 knockouts are lethal, a cell line derived from embryonic TSC2-null Eker rat, named EEF-8, was obtained, as well as TSC2 $2^{-/-}$fibroblasts derived from this model [45]. Mouse embryo fibroblasts (MEFs) isolated from both the TSC1 and TSC 2 knockout mice exhibited constitutive activation of mTOR and S6K1 phosphorylation [46, 47].

In 2001, Arbiser et al. [48] generated a cell line from human sporadic angiomyolipoma of a 63-yearold patient which was not associated with TSC. Cells were transfected with SV40 large T antigen and human telomerase to obtain a stable and immortalized cell line. This cell line expressed tuberin and hamartin, however, it developed an increased activation of MAPK. There was a positive immunohistochemical reaction with cytokeratins which suggests that this culture can represent epithelioid-like cells. This cell line is commercially available [48].

Human cell lines were successfully isolated from tissue obtained from patients with angiomyolipoma in Tuberous Sclerosis Complex. Lesma et al. [49] characterized two cell lines derived from angiomyolipoma of a 42-year-old patient with TSC. Cells were characterized by immunocytochemistry showing high expression of $\alpha$ SMA and HMB-45 antibody immunoreactivity. However, staining with S100, vimentin, CD68 and keratins $8 / 18$ was negative. The second cell population, epithelial-like cells, strongly reacted with the antibody against cytokeratins 8/18 and HMB-45 and did not express $\alpha$-SMA, S-100, vimentin or CD68. Epithelial-like cells $(\mathrm{R}+)$ strongly reacted with RhoA antibody but did not show LOH for the TSC2 gene [49]. Smooth muscle-like cells $(\mathrm{A}+)$ revealed TSC2 LOH and a lack of tuberin expression. A+ cells had receptors for epidermal growth factor (EGF) and insulin-like growth factor 1 (IGF-1) on their surface and EGF medium supplementation was essential for the survival and proliferation of these cells in culture [49]. 
These cell lines were used to study the effects of monoclonal antibody anti-EGFR, rapamycin and other mTOR pathway blockers on cell survival. It was found that anti-EGFR antibody effectively inhibited the proliferation of these cells suggesting its use as an effective therapeutic option [50]. A study of the role of survivin as an inhibitor of apoptosis in these cell lines showed that it may be an important anti-apoptotic and survival factor with the potential of becoming the target of future therapies [51]. Clements et al. [52] demonstrated two cell line populations derived from angiomyolipoma: spindle-shaped cells and epithelioid-like cells. The cultured cells showed constitutive activation of S6K1 protein and strong expression of $\alpha$ SMA. mRNA of gp100, and MART-1 (spindle- shaped cells) and estrogen receptors was detected in these cells by RT-PCR. Cells were cultured in the presence of epidermal growth factor [52] and used to study the role of chemokines in LAM and angiomyolipoma [29] as well as the effects of doxycycline on the proliferation, production of MMPs and adhesion of LAM-related cells [38]. Yu et al. [53] described a line derived from human angiomyolipoma. The cells were spindle-shaped, showed LOH for TSC2, the expression of estrogen receptors and hiperphosphorylation of S6. Both estradiol and, unexpectedly, tamoxifen, stimulated their growth demonstrating that tamoxifen may act as an estrogen agonist in human cultured angiomyolipoma cells [53], in opposition to being an estrogen antagonist in Eker rat-derived ELT3 cells [54]. All human angiomyolipoma cells were used between the second and fourth passage in this experiment [53]. The same, but immortalized human angiomyolipoma cell line, was used to study the level of MMP-2 expression and the mechanism leading to its overexpression [35].

Black et al. [55] studied LAM cells isolated from a fragment of lung tissue using laser micro-dissection. They were immunohistochemically characterized as HMB-45 positive. All cells in this experiment were used between the fourth and eighth passage. The authors found reduced expression of endogenous prostaglandin E2 (PGE2) associated with the decreased expression of the cyclooxygenase 2 (COX2). Production of the VEGF protein family by these cells was documented [55]. Goncharova et al. [56] obtained LAM cell lines from the LAM nodule of lung tissue derived from patients after lung transplantation. Lines were characterized as a-SMA positive, and HMB-45 negative. These cells showed constitutive hyperphosphorylation of p70S6, S6 protein and high proliferative activity, even without growth factor supplementation. Cells were used between the 3rd and 12th passage. This study showed that TSC2 dysfunction and constitutive acti- vation of $\mathrm{mTOR} / \mathrm{S} 6 \mathrm{~K} 1$ attenuates growthinhibitory effect of IFN- $\beta$ and suggests that combination of rapamycin and IFN- $\beta$ cells treatment could abrogate LAM cells proliferation [56]. The same authors have next demonstrated that activation of signal transducer and activator of transcription 3 (STAT3) was essential for the proliferation and survival of this cell line [57].

\section{Conclusions}

LAM cells are a heterogeneous population of cells. Their proliferation leads to the formation of LAM lesions, cysts and lung tissue destruction. Differences in size, morphology and antigen pattern expression, make the identification and isolation of LAM cells very difficult. The phenotype of these cells depends on their microenvironment, exposure to cytokines, growth factors and interactions with other cells. A better understanding of the nature of LAM cells is necessary in order to find an applicable in vitro model, as well as new therapeutic modalities. Analyses of genetic abnormalities or characteristic markers in blood or other body fluids, such as BALF, chyle or urine could provide less invasive methods for early diagnosis of LAM.

\section{Acknowledgment}

This study was supported by grant of Wroclaw Medical University No. Pbmn84.

\section{References}

1. Ryu JH, Moss J, Beck GJ et al. NHLBI LAM Registry Group. The NHLBI lymphangioleiomyomatosis registry: characteristics of 230 patients at enrollment. Am J Respir Crit Care Med. 2006;173:105-111.

2. Taveira-DaSilva AM, Pacheco-Rodriguez G, Moss J. The natural history of lymphangioleiomyomatosis: markers of severity, rate of progression and prognosis. Lymphat Res Biol.2010;8:9-19.

3. Taveira-DaSilva AM, Steagall WK, Moss J. Lymphangioleiomyomatosis. Cancer Control. 2006;13:276-285.

4. Glassberg MK. Lymphangioleiomyomatosis. Clin Chest Med. 2004;25:573-582.

5. Strizheva GD, Carsillo T, Kruger WD, Sullivan EJ, Ryu JH, Henske EP. The spectrum of mutations in TSC1 and TSC2 in women with tuberous sclerosis and lymphangiomyomatosis. Am J Respir Crit Care Med. 2001;163:253-258.

6. Kwiatkowski DJ. Rhebbing up mTOR: new insights on TSC1 and TSC2, and the pathogenesis of tuberous sclerosis. Cancer Biol Ther. 2003;2:471-476.

7. Kristof AS. mTOR signaling in lymphangioleiomyomatosis. Lymphat Res Biol. 2010;8:33-42.

8. Yu J, Parkhitko AA, Henske EP. Mammalian target of rapamycin signaling and autophagy: roles in lymphangioleiomyomatosis therapy. Proc Am Thorac Soc. 2010;7:48-53.

9. Davies DM, de Vries PJ, Johnson SR et al. Sirolimus therapy for angiomyolipoma in tuberous sclerosis and sporadic lymphangioleiomyomatosis: a phase 2 trial. Clin Cancer Res. 2011;17:4071-4081. 
10. Bissler JJ, McCormack FX, Young LR et al. Sirolimus for angiomyolipoma in tuberous sclerosis complex or lymphangioleiomyomatosis. $N$ Engl J Med. 2008;358:140-151.

11. Chachaj A, Drozdz K, Chabowski M et al. Chyloperitoneum, chylothorax and lower extremity lymphedema in woman with sporadic lymphangioleiomyomatosis successfully treated with sirolimus: a case report. Lymphology. 2012;45:53-57.

12. Muzykewicz DA, Sharma A, Muse V, Numis AL, Rajagopal J, Thiele EA. TSC1 and TSC2 mutations in patients with lymphangioleiomyomatosis and tuberous sclerosis complex.JMed Genet. 2009;46:465-468.

13. Carsillo T, Astrinidis A, Henske EP. Mutations in the tuberous sclerosis complex gene TSC2 are a cause of sporadic pulmonary lymphangioleiomyomatosis. Proc Natl Acad Sci U S A. 2000;97:6085-6090.

14. Yu J, Astrinidis A, Henske EP. Chromosome 16 loss of heterozygosity in tuberous sclerosis and sporadic lymphangiomyomatosis. Am J Respir Crit Care Med. 2001;164:1537-1540.

15. Knudson AG. Hereditary cancer: two hits revisited. J Cancer Res Clin Oncol. 1996;122:135-140.

16. Sato T, Seyama K, Fujii H et al. Mutation analysis of the TSC1 and TSC2 genes in Japanese patients with pulmonary lymphangioleiomyomatosis. J Hum Genet. 2002;47:20-28.

17. Goncharova EA, Goncharov DA, Lim PN, Noonan D, Krymskaya VP. Modulation of cell migration and invasiveness by tumor suppressor TSC2 in lymphangioleiomyomatosis. Am J Respir Cell Mol Biol. 2006;34:473-480.

18. Barnes EA, Kenerson HL, Mak BC, Yeung RS. The loss of tuberin promotes cell invasion through the $B$-catenin pathway. Am J Respir Cell Mol Biol. 2010;43:617-627.

19. Matsui K, Tatsuguchi A, Valencia J et al. Extrapulmonary lymphangioleiomyomatosis (LAM): clinicopathologic features in 22 cases. Hum Pathol. 2000;31:1242-1248.

20. Ferrans VJ, Yu ZX, Nelson WK et al. Lymphangioleiomyomatosis (LAM): a review of clinical and morphological features. J Nihon Med Sch. 2000;67:311-329.

21. Krymskaya VP. Smooth muscle-like cells in pulmonary lymphangioleiomyomatosis. Proc Am Thorac Soc. 2008;5:119-126.

22. Matsumoto Y, Horiba K, Usuki J, Chu SC, Ferrans VJ, Moss J. Markers of cell proliferation and expression of melanosomal antigen in lymphangioleiomyomatosis. Am J Respir Cell Mol Biol. 1999;21:327-336.

23. Klarquist J, Barfuss A, Kandala S et al. Melanoma-associated antigen expression in lymphangioleiomyomatosis renders tumor cells susceptible to cytotoxic T cells. Am J Pathol. 2009;175:2463-2472.

24. Zhe X, Schuger L. Combined smooth muscle and melanocytic differentiation in lymphangioleiomyomatosis. $J$ Histochem Cytochem. 2004;52:1537-1542.

25. Logginidou H, Ao X, Russo I, Henske EP. Frequent estrogen and progesterone receptor immunoreactivity in renal angiomyolipomas from women with pulmonary lymphangioleiomyomatosis. Chest. 2000;117:25-30.

26. Matsui K, Takeda K, Yu ZX et al. Downregulation of estrogen and progesterone receptors in the abnormal smooth muscle cells in pulmonary lymphangioleiomyomatosis following therapy. An immunohistochemical study. Am J Respir Crit Care Med. 2000;161:1002-1009.

27. Yu J, Henske EP. mTOR activation, lymphangiogenesis, and estrogen-mediated cell survival: the „perfect storm” of prometastatic factors in LAM pathogenesis. Lymphat Res Biol. 2010;8:43-49.

28. Pacheco-Rodriguez G, Kumaki F, Steagall WK et al. Chemokine-enhanced chemotaxis of lymphangioleiomyomatosis cells with mutations in the tumor suppressor TSC2 gene.J Immunol. 2009;182:1270-1277.

29. Clements D, Markwick LJ, Puri N, Johnson SR. Role of the CXCR4/CXCL12 axis in lymphangioleiomyomatosis and angiomyolipoma. J Immunol. 2010;185:1812-1821.

30. Pacheco-Rodriguez G, Moss J. The role of chemokines in migration of metastatic-like lymphangioleiomyomatosis cells. Crit Rev Immunol. 2010;30:387-394.

31. Hayashi T, Fleming MV, Stetler-Stevenson WG et al. Immunohistochemical study of matrix metalloproteinases (MMPs) and their tissue inhibitors (TIMPs) in pulmonary lymphangioleiomyomatosis(LAM). Hum Pathol. 1997;28:1071-1078 .

32. Zhe X, Jakkaraju S, Schuger L. Tissue inhibitor of metallo-proteinase-3 downregulation in lymphangioleiomyomatosis: potential consequence of abnormal serum response factor expression. Am J Respir Cell Mol Biol. 2003;28:504-511.

33. Zhe X, Yang Y, Schuger L. Imbalanced plasminogen system in lymphangioleiomyomatosis: potential role of serum response factor. Am J Respir Cell Mol Biol. 2005;32:28-34.

34. Odajima N, Betsuyaku T, Yoshida T, Hosokawa T, Nishimura M. High levels of extracellular matrix metalloproteinase inducer are expressed in lymphangioleiomyomatosis. Hum Pathol. 2010;41:935-943.

35. Lee PS, Tsang SW, Moses MA et al. Rapamycin-insensitive up-regulation of MMP2 and other genes in tuberous sclerosis complex 2-deficient lymphangioleiomyomatosis-like cells. Am J Respir Cell Mol Biol. 2010;42:227-234.

36. Moses MA, Harper J, Folkman J. Doxycycline treatment for lymphangioleiomyomatosis with urinary monitoring for MMPs. N Engl J Med. 2006;354:2621-2622.

37. Pimenta SP, Baldi BG, Acencio MM, Kairalla RA, Carvalho CR. Doxycycline use in patients with lymphangioleiomyomatosis: safety and efficacy in metalloproteinase blockade. J Bras Pneumol. 2011;37:424-430.

38. Chang WY, Clements D, Johnson SR. Effect of doxycycline on proliferation, MMP production, and adhesion in LAM-related cells. Am J Physiol Lung Cell Mol Physiol. 2010;299: 393-400.

39. Crooks DM, Pacheco-Rodriguez G, DeCastro RM et al. Molecular and genetic analysis of disseminated neoplastic cells in lymphangioleiomyomatosis. Proc Natl Acad Sci U S A. 2004; 101:17462-17467.

40. Pacheco-Rodriguez G, Steagall WK, Crooks DM et al. TSC2 loss in lymphangioleiomyomatosis cells correlated with expression of CD44v6, a molecular determinant of metastasis. Cancer Res. 2007;67:10573-10581.

41. Cai X, Pacheco-Rodriguez G, Fan QY et al. Phenotypic characterization of disseminated cells with TSC2 loss of heterozygosity in patients with lymphangioleiomyomatosis. Am J Respir Crit Care Med. 2010;182:1410-1418.

42. Kwiatkowski DJ. Animal models of lymphangioleiomyomatosis (LAM) and tuberous sclerosis complex (TSC). Lymphat Res Biol. 2010;8:51-57.

43. Darling TN, Pacheco-Rodriguez G, Gorio A, Lesma E, Walker C, Moss J. Lymphangioleiomyomatosis and TSC2-/cells. Lymphat Res Biol. 2010;8:59-69.

44. Walker CL, Hunter D, Everitt JI. Uterine leiomyoma in the Eker rat: a unique model for important diseases of women. Genes Chromosomes Cancer. 2003;38:349-356.

45. Soucek T, Yeung RS, Hengstschläger M. Inactivation of the cyclin-dependent kinase inhibitor p27 upon loss of the tuberous sclerosis complex gene-2. Proc Natl Acad Sci U S A. 1998; 95:15653-15658. 
46. Kwiatkowski DJ, Zhang H, Bandura JL, Heiberger KM, Glogauer M, el-Hashemite N, Onda H. A mouse model of TSC1 reveals sex-dependent lethality from liver hemangiomas, and up-regulation of p70S6 kinase activity in Tsc1 null cells. Hum Mol Genet. 2002;11:525-534.

47. Zhang H, Cicchetti $\mathrm{G}$, Onda $\mathrm{H}$ et al. Loss of Tsc1/Tsc2 activates mTOR and disrupts PI3K-Akt signaling through downregulation of PDGFR.J Clin Invest. 2003;112:1223-1233.

48. Arbiser JL, Yeung R, Weiss SW et al. The generation and characterization of a cell line derived from a sporadic renal angiomyolipoma: use of telomerase to obtain stable populations of cells from benign neoplasms. Am J Pathol. 2001;159:483-491.

49. Lesma E, Grande V, Carelli S et al. Isolation and growth of smooth muscle-like cells derived from tuberous sclerosis complex-2 human renal angiomyolipoma: epidermal growth factor is the required growth factor. Am J Pathol. 2005;167:1093-1103 .

50. Lesma E, Grande V, Ancona S, Carelli S, Di Giulio AM, Gorio A. Anti-EGFR antibody efficiently and specifically inhibits human TSC2-/-smooth muscle cell proliferation. Possible treatment options for TSC and LAM. PLoS One. 2008;3:3558.

51. Carelli S, Lesma E, Paratore S et al. Survivin expression in tuberous sclerosis complex cells. Mol Med. 2007;13:166-177.
52. Clements D, Asprey SL, McCulloch TA, Morris TA, Watson SA, Johnson SR. Analysis of the oestrogen response in an angiomyolipoma derived xenograft model. Endocr Relat Cancer. 2009;16:59-72.

53. Yu J, Astrinidis A, Howard S, Henske EP. Estradiol and tamoxifen stimulate LAM-associated angiomyolipoma cell growth and activate both genomic and nongenomic signaling pathways. Am J Physiol Lung Cell Mol Physiol. 2004;286: 694-700.

54. Howe SR, Gottardis MM, Everitt JI, Walker C. Estrogen stimulation and tamoxifen inhibition of leiomyoma cell growth in vitro and in vivo. Endocrinology. 1995;136:4996-5003.

55. Black JL, Ge Q, Boustany $\mathrm{S}$ et al. In vitro studies of lymphangioleiomyomatosis. Eur Respir J. 2005;26:569-576.

56. Goncharova EA, Goncharov DA, Chisolm A et al. Interferon beta augments tuberous sclerosis complex 2 (TSC2)-dependent inhibition of TSC2-null ELT3 and human lymphangioleiomyomatosis-derived cell proliferation. Mol Pharmacol. 2008;73:778-788.

57. Goncharova EA, Goncharov DA, Damera G et al. Signal transducer and activator of transcription 3 is required for abnormal proliferation and survival of TSC2-deficient cells: relevance to pulmonary lymphangioleiomyomatosis. Mol Pharmacol. 2009;76:766-777.

Submitted: 4 December, 2012 Accepted after reviews: 12 February, 2013 